\title{
Atomic-scale structure of the $\mathrm{SrTiO}_{3}(001)-c(6 \times 2)$ reconstruction: Experiments and first-principles calculations
}

\author{
C. H. Lanier, ${ }^{1,2}$ A. van de Walle, ${ }^{3}$ N. Erdman, ${ }^{4}$ E. Landree, ${ }^{5}$ O. Warschkow, ${ }^{6}$ A. Kazimirov, ${ }^{7}$ K. R. Poeppelmeier,${ }^{2,8}$ \\ J. Zegenhagen, ${ }^{9,10}$ M. Asta, ${ }^{11}$ and L. D. Marks ${ }^{1,2}$ \\ ${ }^{1}$ Department of Materials Science and Engineering, Northwestern University, Evanston, Illinois 60208, USA \\ ${ }^{2}$ Institute for Catalysis in Energy Processes, Northwestern University, Evanston, Illinois 60208, USA \\ ${ }^{3}$ Engineering and Applied Science Division, California Institute of Technology, Pasadena, California 91125, USA \\ ${ }^{4}$ JEOL USA, Inc., 11 Dearborn Road, Peabody, Massachusetts 01960, USA \\ ${ }^{5}$ RAND Corporation, Arlington, Virginia 22202, USA \\ ${ }^{6}$ School of Physics, The University of Sydney, Sydney, New South Wales 2026, Australia \\ ${ }^{7}$ Cornell High Energy Synchrotron Source, Ithaca, New York 14953, USA \\ ${ }^{8}$ Department of Chemistry, Northwestern University, Evanston, Illinois 60208, USA \\ ${ }^{9}$ Max-Planck Institut für Festkörperforschung, Heisenbergstrasse 1, D-70569 Stuttgart, Germany \\ ${ }^{10}$ European Synchrotron Radiation Facility (ESRF), Boîte Postale 220, F-39043 Grenoble, France \\ ${ }^{11}$ Chemical Engineering and Materials Science Department, University of California at Davis, Davis, California 95616, USA
}

(Received 27 January 2007; revised manuscript received 15 May 2007; published 23 July 2007)

\begin{abstract}
The $c(6 \times 2)$ is a reconstruction of the $\mathrm{SrTiO}_{3}(001)$ surface that is formed between 1050 and $1100{ }^{\circ} \mathrm{C}$ in oxidizing annealing conditions. This work proposes a model for the atomic structure for the $c(6 \times 2)$ obtained through a combination of results from transmission electron diffraction, surface x-ray diffraction, direct methods analysis, computational combinational screening, and density functional theory. As it is formed at high temperatures, the surface is complex and can be described as a short-range-ordered phase featuring microscopic domains composed of four main structural motifs. Additionally, nonperiodic $\mathrm{TiO}_{2}$ units are present on the surface. Simulated scanning tunneling microscopy images based on the electronic structure calculations are consistent with experimental images.
\end{abstract}

DOI: 10.1103/PhysRevB.76.045421 PACS number(s): 68.35.Bs, 71.15.Mb, 61.10.Nz, 68.37.Ef

\section{INTRODUCTION}

Strontium titanate $\left(\mathrm{SrTiO}_{3}\right)$ has received considerable attention over the last decade because of its numerous technological applications, ${ }^{1}$ including use as a substrate for thin film growth ${ }^{2}$ and as a candidate crystalline gate dielectric in silicon-based devices. ${ }^{3,4}$ Furthermore, the surface of $\mathrm{SrTiO}_{3}$ plays an important role in surface reactions and catalysis. ${ }^{5,6}$ Many of these applications are governed by interfacial processes, which motivates a continuing interest in the surfaces of $\mathrm{SrTiO}_{3}$, but despite extensive research into the surface properties, there remain many important unanswered questions. Only recently, the $(2 \times 1)$ and $c(4 \times 2)$ reconstructions on $\mathrm{SrTiO}_{3}(001)$ have been solved by direct methods. ${ }^{7,8}$ Other reconstructions have been observed on pure $\mathrm{SrTiO}_{3}(001)$, including the $(1 \times 1),(2 \times 2), c(2 \times 2)$, $(4 \times 4), \quad c(4 \times 4), \quad(6 \times 2), \quad c(6 \times 2), \quad(\sqrt{5} \times \sqrt{5}) R 26^{\circ}, \quad$ and $(\sqrt{13} \times \sqrt{13}) R 33.7^{\circ} .{ }^{6,9-19}$ Models have been proposed for many of these structures, though they are often inconsistent with one another, and theoretical models have also been developed; however, these too remain contradictory. ${ }^{18,20-22}$

One structure which has proven especially difficult to determine is the $\mathrm{SrTiO}_{3}(001)-c(6 \times 2)$ surface reconstruction. The main challenge, as will be shown, is the fact that a single reconstruction is unable to adequately describe the surface, which probably is related to the high annealing temperature $\left(1050-1100{ }^{\circ} \mathrm{C}\right)$ required to form the surface. Instead, the equilibrium $c(6 \times 2)$ surface at the formation temperature is found to be short-range ordered, consisting of microscopic domains of four related structural motifs. Upon sufficiently rapid cooling, the surface structure is quenched, and the domains of the four motifs persist.

The $c(6 \times 2)$ has been previously reported by Jiang and Zegenhagen with scanning tunneling microscopy (STM) and low-energy electron diffraction ${ }^{23,24}$ and by Naito and Sato with reflection high-energy electron diffraction (RHEED). ${ }^{17}$ The STM results are included here, and newly available x-ray diffraction results are also utilized. The $c(6 \times 2)$ studied by RHEED ${ }^{17}$ was found to coexist with domains of $(\sqrt{13} \times \sqrt{13}) R 33.7^{\circ}$ and may likely be different from the surface studied here, as the surface preparation, which is known to play a large role, was different. As mentioned earlier, a $(6 \times 2)$ overlayer has also been observed on $\mathrm{Nb}$ doped $\mathrm{SrTiO}_{3}$ (001) (Ref. 19); however, this structure is not the same as the $c(6 \times 2)$ reported here since the $(6 \times 2)$ surface unit cell is not centered and thus has a different symmetry and structure.

Direct methods for surfaces based on diffraction data have been employed to solve numerous structures (for more information, see Refs 25 and 26), including two other surface reconstructions on $\mathrm{SrTiO}_{3}(001)$, the $(2 \times 1)$ and $c(4 \times 2), 7,8$ as well as the $\mathrm{SrTiO}_{3}$ (106) surface ${ }^{27}$ and the $(\sqrt{5} \times \sqrt{5}) R 26.6^{\circ}$ reconstruction on $\mathrm{LaAlO}_{3}(001) .{ }^{28}$ In some cases, e.g., the $(2 \times 1)$ and $c(4 \times 2)$ reconstructions on $\mathrm{SrTiO}_{3}$, direct methods can be used to find all of the atoms in the surface structure. However, even with ideally perfect data, sometimes direct methods fail to resolve the atomic positions of certain atoms, particularly weakly scattering elements. Moreover, if disorder or twinning is present on the surface, structure completion (finding the full structure from 
an initial fragment) becomes exceedingly difficult. In this work on the $c(6 \times 2)$ reconstruction, direct methods alone did not result in a structure solution, but instead a combinatorial approach was taken that merged a variety of experimental and computational techniques and resulted in a model of the $\mathrm{SrTiO}_{3}(001)-c(6 \times 2)$ surface that is consistent with all available experimental reports.

In more detail, the approach used in this work is to apply direct methods on a set of transmission electron and x-ray diffraction data ${ }^{25,26}$ in order to determine the approximate positions of the surface cations. Since the weak scattering of oxygen ions prevented conclusive determination of their positions from diffraction methods alone, computational combinatorial screening methods were used along with firstprinciples calculations to identify candidate oxygen configurations. First principles calculations were also used to more accurately determine the surface cation positions. These structural configurations were then used as input for structure refinement using surface $\mathrm{x}$-ray data with the help of the SHELX-97 (Ref. 29) program, and simulated STM images from the output of the $a b$ initio calculations were also compared with available experimental STM images as a final cross-check. The proposed surface structure for the $c(6 \times 2)$ reconstruction is consistent with all of the available experimental and computational evidence.

\section{EXPERIMENTAL AND COMPUTATIONAL METHODS}

Transmission electron diffraction (TED) experiments were conducted on samples prepared from single crystal, undoped $\mathrm{SrTiO}_{3}(001)$ wafers $\left(10 \times 10 \times 5 \mathrm{~mm}^{3}, 99.95 \%\right.$ pure $)$. The wafers were cut into $3 \mathrm{~mm}$ diameter disks using a rotary disk cutter, mechanically thinned to $\sim 120 \mu \mathrm{m}$, polished, dimpled, and ion milled to electron transparency with a $4.8 \mathrm{kV} \mathrm{Ar}^{+}$ion beam. Samples were annealed for $2-5 \mathrm{~h}$ in a tube furnace at $1050-1100{ }^{\circ} \mathrm{C}$ under a flow of high purity oxygen at atmospheric pressure in order to produce the reconstructed surface. Transmission electron microscopy images and off-zone diffraction patterns were obtained on the Hitachi ultrahigh vacuum (UHV) H9000 electron microscope, operated at $300 \mathrm{kV}$. A series of off-zone diffraction patterns were recorded with exposure times ranging from 0.5 to $120 \mathrm{~s}$. The negatives were scanned with a $25 \mu \mathrm{m}$ pixel size and digitized to 8 bits with an Optronics P-1000 microdensitometer. The diffraction intensities were then averaged with the $c 2 \mathrm{~mm}$ Patterson plane group symmetry, yielding 58 independent intensities.

Surface x-ray diffraction (SXRD) experiments were performed at the BW2 wiggler beamline at the Hamburg Synchrotron radiation laboratory using radiation of $8 \mathrm{keV}$, monochromatized and sagittally focused by a pair of $\mathrm{Si}(111)$ crystals. Two single crystal $\mathrm{SrTiO}_{3}(001)$ samples were annealed at the Max-Planck Institut in Stuttgart at $1100{ }^{\circ} \mathrm{C}$ in flowing oxygen for about $2 \mathrm{~h}$. The samples were stored in an oxygen atmosphere container and shipped to another laboratory where they were characterized at room temperature by SXRD in air. One of the samples was measured in air a few days after the preparation. The second crystal was reloaded into a UHV chamber, exposed to a mild annealing in UHV at $\sim 300{ }^{\circ} \mathrm{C}$, and loaded into a small portable UHV chamber which was mounted on the diffractometer for the SXRD measurements. The acquisition of the diffraction data took approximately 3 days for each of the two samples. The stability of the surface over the acquisition period was ascertained by checking the stability of the (080) reflection at regular intervals, and integrated intensities were recorded for 263 in-plane reflections and 32 rods. The data were corrected for footprint and polarization, had reflections below the critical angle discarded, and were averaged using $C 2 \mathrm{~mm}$ space group symmetry. The data taken for the two differently handled samples (oxygen annealed and oxygen and UHV annealed) were used separately for the structure refinement. See Ref. 30 for a copy of the SXRD data.

STM images were obtained using an Omicron "microSTM" system operating under UHV conditions. The $\mathrm{SrTiO}_{3}(001)-c(6 \times 2)$ sample, which was prepared outside the system by annealing at $1100{ }^{\circ} \mathrm{C}$ in a flow of oxygen, was loaded into the UHV-STM system and annealed for approximately $10-15 \mathrm{~min}$ at $800^{\circ} \mathrm{C}$ in order to generate enough oxygen vacancies in the bulk to allow imaging by STM. Tungsten tips were used, and the STM scanner was calibrated with the use of the well-known $\operatorname{Si}(111)-(7 \times 7)$ reconstruction. Images were obtained in constant current topography mode, and the sample was biased positively with respect to the tip, thus tunneling occurred into the empty states of the sample.

Direct methods were used to determine the scattering potential map of the surface structure based on the transmission electron and x-ray diffraction data. Direct methods solve the phase problem by utilizing probability relationships between the amplitude and the phase of the diffracted beams. A set of phases is determined with the lowest figures of merit most consistent with scattering from discrete atoms and is combined with the measured beam amplitudes. By this approach, scattering potential maps and candidate structures can be generated from the diffraction data without the need for a structure guess.

First-principles $(a b$ initio $)$ density functional theory (DFT) calculations were performed using the Vienna $a b$ initio simulation package (VASP), ${ }^{31-34}$ which solves the DFT equations within the plane-wave-pseudopotential formalism. The $\mathrm{SrTiO}_{3}(001)$ surface was represented by a surface slab model as illustrated in Fig. 1, with all atomic positions relaxed except for the center atomic layer which was held fixed at bulk positions and lattice parameters [determined in a separate bulk local density approximation (LDA) calculation]. The calculated lattice parameter $(3.827 \AA)$ is about $2 \%$ smaller than the experimental lattice parameter at room temperature $(3.905 \AA)$, which is typical for LDA calculations. Core electrons were represented by Vanderbilt-type ultrasoft pseudopotentials ${ }^{35,36}$ (VASP library pseudopotentials "Ti," "Sr," and "O_s"), and electron exchange and correlation were treated in the LDA (Ceperley and Adler ${ }^{37}$ ). The plane-wave basis set was cut off at $270 \mathrm{eV}$. Simulated STM images were produced from the output of the $a b$ initio calculations in the Tersoff-Hamann approximation, ${ }^{38}$ which assumes that the pointlike STM tip follows an isosurface of the local density of states within a specified energy window around the Fermi level. A relatively high isodensity surface lying very close to 

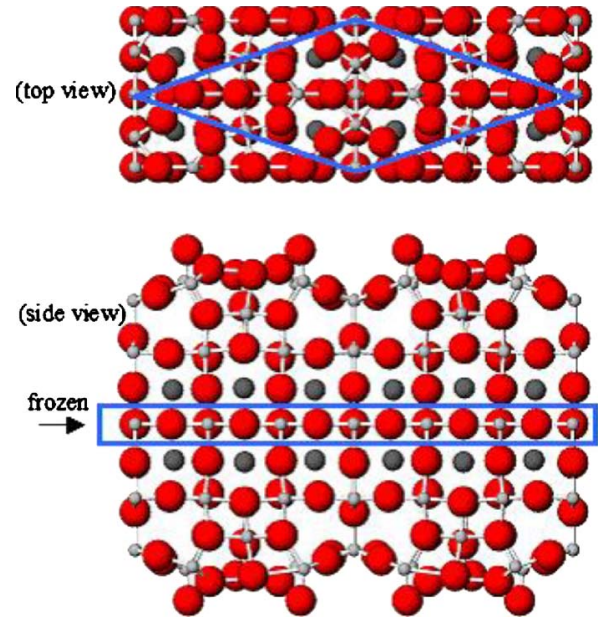

FIG. 1. (Color online) Geometry employed in the $a b$ initio calculations, with the primitive $c(6 \times 2)$ surface unit cell outlined (representative structure shown). Large red spheres are oxygen, small light gray spheres are titanium, and medium dark gray spheres are strontium. The geometries of the two lowest-energy structures at each composition were also reoptimized using a thicker slab (including four strontium layers instead of two) in which the middle layer (containing $\mathrm{Ti}$ and $\mathrm{O}$ ) was kept frozen.

the surface was used, thus enabling us to use a smaller "vacuum" region in the supercell calculation. Simulated images were created using the integrated density of unoccupied states between 0 and $+2.1 \mathrm{~V}$ relative to the Fermi level.

Surface $\mathrm{X}$-ray diffraction data structure refinements were performed using the SHELX-97 code ${ }^{29}$ which is a widely used structural refinement program used in many fields including crystallography. The atomic positions for each of the plausible structures generated by DFT were input into the SHELX97 program and refined primarily against the experimental data obtained in air. Since LDA calculations underestimate the lattice parameters, we scaled all atomic positions isotropically until the calculated lattice parameters matched the experimental value. This approach is preferable to imposing the experimental in-plane lattice parameters in the calculations, since the system would then contract perpendicular to the surface, resulting in an unphysical distortion that would be difficult to correct. The data were decomposed into 33 batches, 1 for the in-plane set and 32 for each of the rods, and each batch was given a different scale factor to account for experimental error in the data collection owing to changes in the sample-detector geometries upon measurements of different rods. Refinement parameters are given in the input (.ins) file and are described in the SHELX-97 manual. See Ref. 30 for a copy of the input (.ins) file and final $\left(F_{c}\right)^{2}$ values.

\section{RESULTS}

\section{A. Transmission electron microscopy}

Dark field transmission electron microscopy images and off-zone diffraction patterns were obtained for the $c(6 \times 2)$ surface, as shown in Fig. 2. For the sample preparation tech-

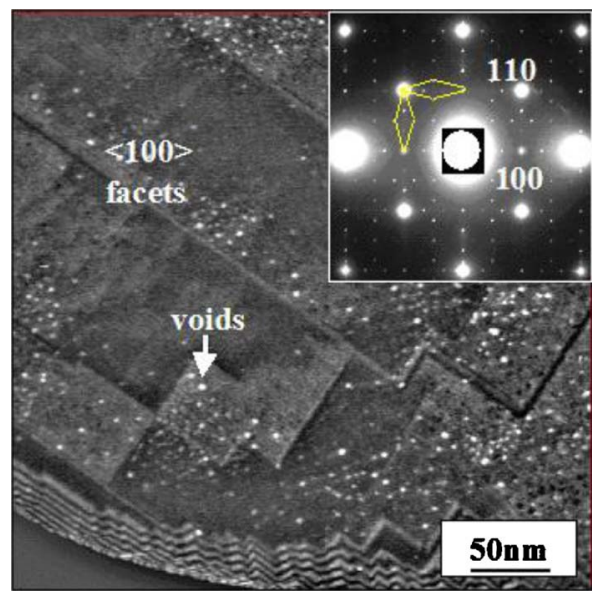

FIG. 2. (Color online) Dark field image and transmission electron diffraction data (inset) from the $c(6 \times 2)$ surface. Primitive reciprocal unit cells for the two surface domains unit cell are outlined. Adapted from Ref. 39.

niques employed here, the $c(6 \times 2)$ surface reconstruction is highly reproducible and was found to be air stable over a period of months. The dark field image in Fig. 2 shows a flat, faceted surface with large terraces separated by step bunches, and the $c(6 \times 2)$ surface reconstruction was found to cover the entire surface. Voids are also visible in the near-surface region of the sample, and similar morphologies have been observed for other reconstructed $\mathrm{SrTiO}_{3}$ (001) surfaces. ${ }^{39}$

\section{B. Direct methods}

Direct methods provided the scattering potential maps shown in Fig. 3 based on surface x-ray diffraction data [Figs. 3(a)-3(c)] and transmission electron diffraction data [Fig. $3(\mathrm{~d})$ ]. Further analysis, based on symmetry and difference maps, indicated that the dark spots were titanium atom sites and that the surface contained no strontium atoms. Numerous attempts were made to refine a single structure with reasonable oxygen sites, but no single structure yielded good results. This occurred, as will be shown, because the surface is really a mixture of four different structural motifs. While the positions of the titanium atoms averaged over the four structural motifs could be determined in projection from the electron diffraction data and in three dimensions from the $\mathrm{x}$-ray diffraction data, the positions of the surface oxygen atoms could not be determined owing to larger variation of the oxygen positions among the four motifs. This conclusion was reached by applying a combinational screening method in conjunction with first-principles methods to identify plausible oxygen configurations, with the averaged positions of the titanium atoms from the direct methods analysis used as the input for the screening method.

\section{Computational screening}

The determination of the minimum energy oxygen configuration represented a challenging optimization problem, given the large configuration space that needed to be sampled and the presence of an enormous number of local 


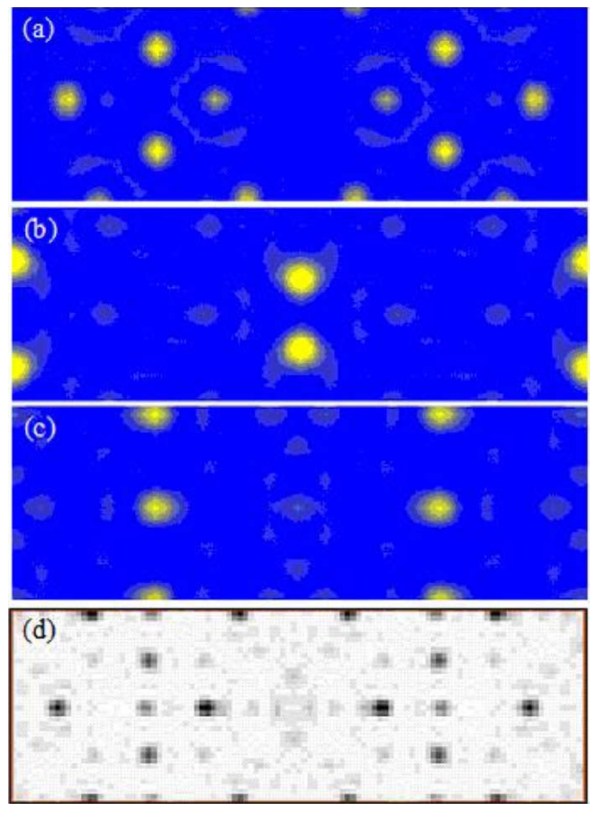

FIG. 3. (Color online) [(a)-(c)] Electron density maps for the centered $c(6 \times 2)$ unit cell from SXRD direct methods at $z=3.6 \AA$, $z=2.8 \AA$, and $z=2.0 \AA$ above the first bulklike $\mathrm{TiO}_{2}$ layer, respectively. Regions of high electron density (possible atomic sites) are yellow (light). (d) Scattering potential map (projected) for the centered $c(6 \times 2)$ unit cell from TED direct methods. Regions of high scattering potential (possible atomic sites) are black.

minima in the system's potential energy surface, i.e., the energy of the system as a function of all atomic coordinates. Each local minimum is surrounded by a basin of attraction, where the set of all points connected to that local minimum follows a continuous path along which the energy decreases. The screening approach divided the optimization problem into (i) a discrete outer optimization problem over the different basins of attraction and (ii) a continuous inner optimization problem over atomic coordinates within each basin. The outer optimization problem scanned over basins and provided suitable starting points for the inner continuous optimization problem. In effect, each basin was represented by the selection of one starting point or starting configuration within it. The specific starting configuration in a basin was somewhat arbitrary since the inner continuous optimization problem should find the same local minimum regardless of the starting configuration used.

Starting configurations were constructed via enumeration of every possible placement combination of oxygen atoms on a lattice of plausible candidate sites. These candidate sites, shown in Fig. 4, are located at the midpoint of (1) every pair of titanium atoms separated by $\leqslant 4.25 \AA$ and $(2)$ every triplet of titanium atoms separated by $\leqslant 4.25 \AA$. Fourcoordinated oxygen sites were not considered, because they either produced redundant sites or required at least one of the four titanium-oxygen bonds to be longer than $2.3 \AA$. Onefold coordinated oxygen sites on top of each of the four symmetrically distinct surface titanium atoms were considered as well.

The total number of possible ways to place oxygen atoms on the candidate sites was $2^{40}$, as there were 40 candidate

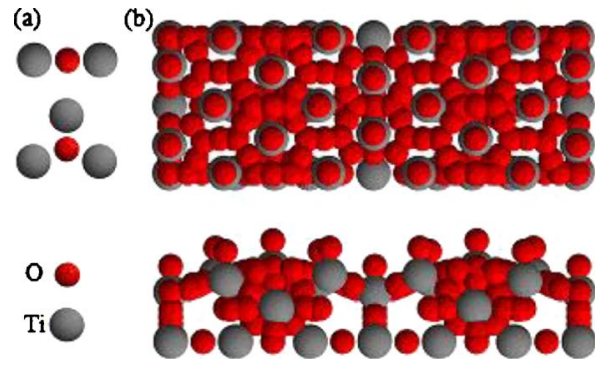

FIG. 4. (Color online) (a) Geometric rules used to generate candidate $\mathrm{O}$ sites shown in (b). The top panel is view toward the surface, while the bottom panel is a side view with the free surface pointing upward.

oxygen sites in the asymmetric unit of the surface unit cell. All of these configurations possess, by construction, the $C 2 \mathrm{~mm}$ space group determined from the experimental SXRD data. Since it would have been prohibitively computationally expensive to calculate the minimum energy within each basin associated with each of these starting configurations via first-principles methods, a hierarchy of increasingly precise criteria was utilized instead to screen out high-energy configurations.

At the coarsest level a simple geometric criteria was used, discarding configurations (1) with an oxygen deficiency exceeding two oxygen atoms per primitive surface unit cell, (2) with oxygen-oxygen bonds shorter than $1.8 \AA$, or (3) containing a titanium atom with a coordination number less than 3 or more than 6 . These simple criteria reduced the number of plausible configurations to 17095 . While this number remained too large to be handled via ab initio methods, it was easily manageable using a simple electrostatic pair potential model, where the species $\mathrm{Sr}, \mathrm{Ti}$, and $\mathrm{O}$ take the nominal charges $2+, 4+$ and $2-$, respectively, which could be used to efficiently identify the most promising configurations.

The electrostatic energy was calculated for each of the 17095 candidate starting configurations previously identified. Note that the atomic positions were not relaxed in these calculations, otherwise the system would have collapsed to a point, since there were no short-range repulsive components in the interatomic forces. For nonstoichiometric structures (stoichiometry of $\mathrm{TiO}_{2-x}$, where $x>0$ ), the charges of all titanium atoms were reduced to $4-2 x$ to maintain charge balance, since the Fermi level would lie within the titanium bands under oxygen-deficient conditions.

At the end of this screening step, $\sim 75$ structures with the lowest electrostatic energy were retained, at each of the three surface stoichiometries considered (from zero to two oxygen vacancies per primitive surface unit cell). Fully relaxed LDA calculations were then performed for each of these $\sim 75$ structures using the VASP code. A representative structural geometry is illustrated in Fig. 1. Given the large number of candidate geometries, a relatively thin slab was used to represent the surface and the Brillouin zone was sampled at the $\Gamma$ point only, in order to limit the computational costs. After the structural relaxations, some of the starting configurations that were initially distinct actually converged to the same configuration: the $\sim 75$ starting configurations produced 64 distinct relaxed geometries. The convergence of some of the 

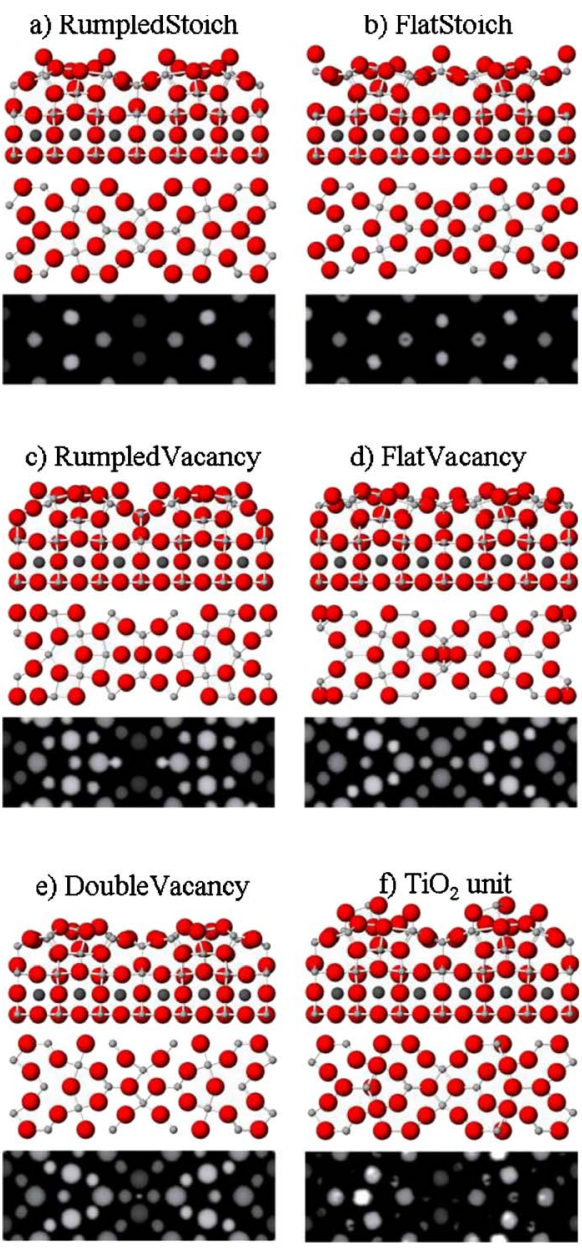

FIG. 5. (Color online) Candidate surface reconstructions showing side view, top view (showing only atoms in the topmost surface layer), and simulated STM image. Large red spheres are oxygen, small light gray spheres are titanium, and medium dark gray spheres are strontium.

configurations toward each other was an indication that the initial partitioning of configuration space was sufficiently fine, as the "distance" between any two starting configurations was, on average, slightly smaller than the size of the typical basin of attraction.

The lowest-energy configurations, i.e., structural motifs, thus identified for each of the three stoichiometries are shown in Fig. 5. These geometries were reoptimized using a thicker slab (twice the thickness shown in Fig. 1) and a finer $k$-point mesh $(4 \times 4 \times 1)$ to yield more accurate energies. At the $\mathrm{TiO}_{2}$ stoichiometry, the lowest-energy structure is labeled as "RumpledStoichiometric" [Fig. 5(a)]. The next lowestenergy structure, labeled as "FlatStoichiometric" [Fig. 5(b)], is $0.37 \mathrm{eV}$ less stable (per primitive unit cell).

At an oxygen content corresponding to one oxygen vacancy per primitive surface unit cell, the screening algorithm identified the "RumpledVacancy" as the lowest-energy structure [Fig. 5(c)]. Visual inspection of that structure revealed that slight displacements along the surface normal of the titanium atoms near the center of the cell changed their coordination from fourfold to fivefold, resulting in another

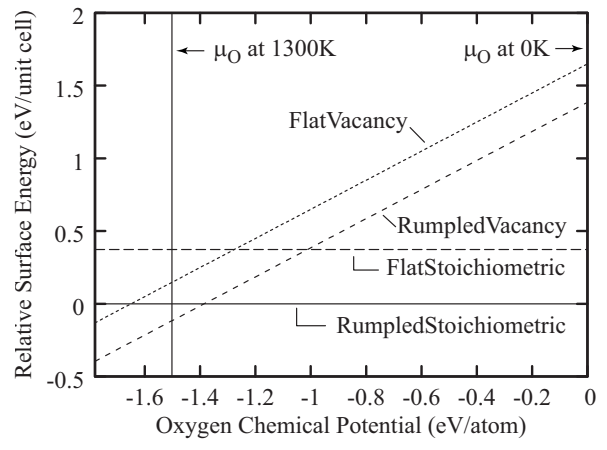

FIG. 6. Relative surface energy per primitive surface unit cell of the four proposed surface motifs as a function of oxygen chemical potential. The surface energies are given relative to the RumpledStoichiometric structure and the chemical potential is relative to its value at $0 \mathrm{~K}$.

plausible structure, labeled "FlatVacancy" [Fig. 5(d)]. Given that the positions determined from direct methods were averaged over the four structural motifs, it is not entirely surprising that some adjustments would be required for the cation positions of any one of the individual structures. While the combinational screening did not find this structure automatically, as it assumed the positions of the $\mathrm{Ti}$ atom to be exact, it did identify a sufficiently similar structure to enable its discovery. During our initial lower-precision screening, the FlatVacancy structure appeared to have a lower energy than the RumpledVacancy structure. However, our more accurate reoptimization of the geometries revealed that the RumpledVacancy structure is the ground state at that composition, with an energy $0.26 \mathrm{eV} /$ unit cell lower than the FlatVacancy structure.

At the composition corresponding to two oxygen vacancies per primitive surface unit cell, the "DoubleVacancy" structure was identified as the lowest-energy structure [Fig. $5(\mathrm{e})]$. The second most stable structure is more than $3 \mathrm{eV} /$ unit cell less stable than the DoubleVacancy structure and can thus be ruled out.

The relative surface energy per primitive unit cell for each of these structural motifs was calculated and plotted as a function of oxygen chemical potential in Fig. 6. It is noted that the DoubleVacancy structure has a potential that is so high that its corresponding line lies far above the range of the figure and is therefore unlikely to be present on the surface. Since the exact surface energies are also a function of the $\mathrm{Ti}$ and $\mathrm{Sr}$ chemical potentials (which are difficult to infer from experimental conditions), we plot the surface energies relative to the RumpledStoichiometric surface energy. This difference in surface energies is sufficient to assess the relative stability of the motifs and offers the advantage that the contributions of the $\mathrm{Ti}$ and $\mathrm{Sr}$ chemical potentials cancel out exactly (because all motifs have the same number of $\mathrm{Ti}$ or $\mathrm{Sr}$ atoms). In contrast, the dependence on the $\mathrm{O}$ chemical potential cannot be similarly eliminated because the different motifs have different oxygen contents.

The range of chemical potentials considered corresponds to temperatures ranging from 0 to $1300 \mathrm{~K}$. The oxygen chemical potential (in $\mathrm{O}_{2}$ at atmospheric pressure) was obtained from the equation 


$$
\mu_{\mathrm{O}}(T)=(1 / 2) \mu_{\mathrm{O}_{2}}(T)=(1 / 2)\left[H_{\mathrm{LDA}}+H(T)-H(0)-T S(T)\right],
$$

where $H_{\mathrm{LDA}}=-9.676 \mathrm{eV}$ (from a LDA calculation of an isolated $\mathrm{O}_{2}$ molecule) and the following tabulated thermodynamic values from Ref. 40 were used: $H(1300 \mathrm{~K})$ $=33344 \mathrm{~J} / \mathrm{mol}, \quad H(0 \mathrm{~K})=-8683 \mathrm{~J} / \mathrm{mol}, \quad$ and $S(1300 \mathrm{~K})$ $=252.878 \mathrm{~J} /(\mathrm{mol} \mathrm{K})$.

It is expected that the actual relative surface energies can be read off from Fig. 6 at a value of the oxygen chemical potential lying somewhere between the calculated extremes shown in the figure. At $T=0 \mathrm{~K}$, the calculations have assumed zero entropy and therefore overstabilize the stoichiometric phases, while at $T=1300 \mathrm{~K}$, the calculations only account for the entropy of the gas phase and, since the free energy change of the solid phases may partially offset the $\mathrm{O}_{2}$ chemical potential change, probably result an overstabilization of the gas phase and of the nonstoichiometric phases.

The surface energies of the four structural motifs considered (RumpledStoichiometric, FlatStoichiometric, FlatVacancy, and RumpledVacancy) lie within $0.4 \mathrm{eV} /$ unit cell of each other for chemical potentials slightly below the $1300 \mathrm{~K}$ value. The actual energy range is likely to be even smaller than our calculated range of $0.4 \mathrm{eV}$ because our results neglect the contribution of lattice vibrations to the free energy. Structures that are very stable (low in energy) tend to be stiffer and therefore have a lower vibrational entropy and a more positive free energy. Conversely, vibrational effects tend to lower the free energies of high-energy structures, resulting in a reduction of the spread in the free energies. Thus, the surface energy differences lie in a range that is likely to be somewhat smaller than $0.4 \mathrm{eV}$, and thus comparable in magnitude to $k_{B} T$ at $1300 \mathrm{~K}$ (about $0.12 \mathrm{eV}$ ), making it quite plausible for the equilibrium surface structure to consist of a disordered mixture of these four structural motifs.

The accuracy of the approach we used to obtain the oxygen chemical potential is limited by the fact that the LDA tends to poorly predict the energy of an isolated molecule. An alternative approach, following Ref. 20, that avoids calculating isolated molecule energies yielded qualitatively similar conclusions: All points where the different surface energies intersect lie between the values of the $\mathrm{O}$ chemical potential at 0 and $1300 \mathrm{~K}$.

The four structural motifs RumpledStoichiometric, FlatStoichiometric, FlatVacancy, and RumpledVacancy can be described using four atomic layers. Starting at the bottom for all motifs (in reference to the geometry shown in Fig. 5), there is a bulklike $\mathrm{TiO}_{2}$ layer followed above with a bulklike $\mathrm{SrO}$ layer, and these two layers are nearly identical in all four motifs. The next $\mathrm{TiO}_{2}$ layer up is similar in all structural motifs and has a rumpled bulklike structure, with relaxations along the direction normal to the surface of at most $\sim 0.12 a_{\text {bulk }}$. Finally, the topmost layer is different for each of the four motifs in the number and placement of the oxygen atoms: the top layer has a $\mathrm{Ti}_{20} \mathrm{O}_{40}$ stoichiometry in the stoichiometric structure centered unit cell and has a $\mathrm{Ti}_{20} \mathrm{O}_{38}$ stoichiometry in the vacancy structure centered unit cell. Note that the titanium positions are nearly identical in all

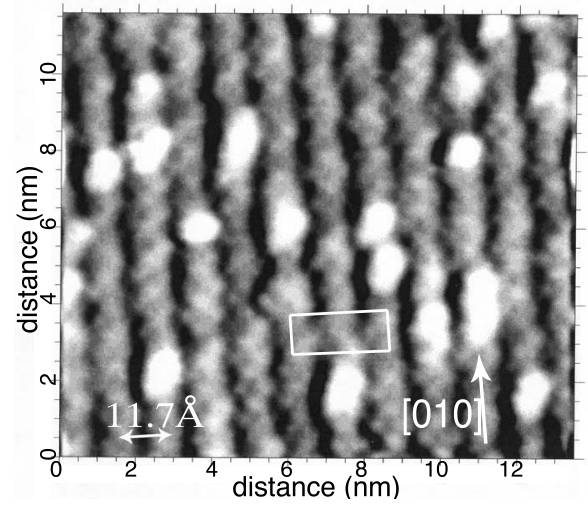

FIG. 7. High resolution STM image of the $c(6 \times 2)$ surface reconstruction $\left(V_{s}=2.1 \mathrm{~V}, I=0.28 \mathrm{nA}\right)$. The $c(6 \times 2)$ centered unit cell is outlined. Adapted from Ref. 23.

structures. See Ref. 30 for the atomic positions of the four structural motifs.

For each structural motif, the topmost layer contains a zigzag along the $b$ (short axis) direction of fivefold coordinated titanium atoms in the form of truncated octahedra. In the centered unit cell, the two zigzags are located at approximately $\frac{1}{4}$ and $\frac{3}{4}$ along the length of the long $(a)$ axis (see Fig. 5), and the relative orientation of the truncated octahedra along the zigzags is the same for three of the four structures and is reversed in the RumpledVacancy structure. In the rumpled structures (RumpledStoichiometric and RumpledVacancy), the zigzag is elevated normal to the surface relative to the center of the unit cell, and in the flat structures (FlatStoichiometric and FlatVacancy), the center of the unit cell is at approximately the same elevation as the zigzag. Accordingly, the titanium atoms at the center of the unit cell (not part of the zigzag) in the rumpled structures are coordinated to the bulklike layer below, while in the flat structures they are not. The coordination of the titanium atoms at the center of the cell is the driving force for the placement of the singly coordinated oxygen (if any) in the various structures. In the structures containing a singly coordinated oxygen, i.e., the RumpledStoichiometric, FlatStoichiometric, and RumpledVacancy structures, the singly coordinated Ti-O bonds are $1.68,1.65$, and $1.65 \AA$ long, respectively, indicating double bond (titanyl) character. Essentially, the differences among the four structures lie in the relative orientation of the truncated octahedra in the zigzag chain, the elevation and coordination of the titanium atoms located in the center of the unit cell, and the placement of the singly coordinated oxygen (if any) at the surface.

\section{STM experiment and simulation}

In STM images taken under empty-state bias conditions (Fig. 7), the $c(6 \times 2)$ reconstruction appears as bright rows with a spacing of $11.7 \AA$ [cf. with $11.715 \AA$ for $\frac{1}{2}$ the $c(6$ $\times 2)$ long axis length, $23.43 \AA$ ]. Confirmed to be $c(6 \times 2)$ by low-energy electron diffraction, the reconstruction was found to cover the surface uniformly wherever probed by the STM. In large-scale images (not shown), the rows appear to be 
aligned with equal probability along the [100] or [010] crystal directions, and in addition to the rows, bright protrusions situated on the rows can be seen randomly distributed over the surface with a density of approximately one for every three $c(6 \times 2)$ centered unit cells. It is noted that sufficient conductivity in $\mathrm{SrTiO}_{3}$ is achieved with an overall carrier density due to oxygen vacancies smaller than $10^{18} \mathrm{e} / \mathrm{cm}^{3}$, i.e., roughly 1 out of every 30 neighboring oxygen atoms missing. It is expected that the density of oxygen vacancies on the surface may be slightly higher, but still low compared to the density of observed contrast variations. Furthermore, preliminary experimental studies in which SXRD data were collected on samples used for STM and low-energy electron diffraction have evidenced that the UHV anneal prior to STM measurements has a minimal effect on the $c(6 \times 2)$ structure.

The simulated STM images, shown in Fig. 5 for each of the structural motifs considered, confirm that in empty state only titanium atoms image brightly, while oxygen atoms are dark, and thus the experimentally observed rows are, in fact, the zigzags of truncated octahedra discussed earlier. Note that the pointlike tip approximation and the tracing of a relatively high isodensity surface resulted in simulated STM images of higher resolution (sharper) than the experimental image. Upon detailed investigation of the experimental image, changes in the relative orientation of the zigzags can be seen occasionally from one row to another, evidence of domain boundaries between different structural motifs.

Upon inspection of the simulated STM images from the structural motifs alone, the bright protrusions observed in the experimental STM images are not accounted for. Based on the previous observation that the STM is imaging titanium atoms, it was determined that the contrast of the bright protrusion is due to excess nonperiodic titanium atoms along the zigzag. Upon studying plausible structures, a likely location for the titanium atom is readily apparent in the RumpledStoichiometric structure. This plausible geometry is suggested by the fact that the two singly coordinated oxygen atoms are at just the right position so that an additional $\mathrm{TiO}_{2}$ unit could be placed on the surface, and the inserted titanium atom would have a fourfold coordination and the inserted oxygen atoms would complete the octahedral coordination of the truncated octahedra in the zigzag. To clarify the nature of these bright protrusions, a simulated STM image was generated of the RumpledStoichiometric surface with an additional $\mathrm{TiO}_{2}$ unit located on the zigzag [see Fig. 5(f)], and the calculated STM image of this surface is in qualitative agreement with the experimentally observed bright protrusions. Note that the final surface stoichiometry is $\mathrm{Ti}_{21} \mathrm{O}_{42}$ for one unit added per centered unit cell, and thus $\mathrm{TiO}_{2}$ is added to the structure in a stoichiometric manner. See Ref. 30 for the DFT refined positions of the $\mathrm{TiO}_{2}$ unit.

\section{E. Structure refinement}

To substantiate the proposed $c(6 \times 2)$ surface structure model, refinement with XRD data was carried out by means of the SHELX-97 refinement program. ${ }^{29}$ Use of this program allowed for the refinement of the complicated, multido- mained $c(6 \times 2)$ structure through partial occupancies of atom sites. Figures of merit including weighted $R$ values $\left(w R^{2}\right)$ and goodness of fit were employed as a gauge for the quality of the refinement, and the Hamilton $R$-factor ratio ${ }^{41}$ was utilized to compare $w R^{2}$ values for structural refinements with various numbers of parameters. The absolute values of the figures of merit do not hold much meaning outside of this study, as this is not a standard SHELX structural refinement, but rather the figures of merit are used to compare models relative to one another. Further, it is important to note that one should not expect a perfect fit between the DFTcalculated positions and the refined positions. Both methods invoke approximations: notably, the refinement process relies on partial occupancies to model disorder, and the DFT calculations neglect thermal expansion, which could affect the average positions of atoms in low-symmetry environments and have an accuracy limited by the unavoidable approximation of the exchange-correlation functional and, to a lesser extent, by the finite $k$-point mesh and energy cutoff.

The four DFT-relaxed structural motifs were refined independently for 25 least squared cycles, and the structures had three bulklike layers below the surface atoms, as illustrated in the cartoons of Fig. 5. Additionally, in order to better represent the surface from which the data were acquired, all four structural motifs were combined and refined simultaneously for 25 least squared cycles. In this case, the combined structure had the same three bulklike layers as the other structures but had a surface containing the atoms from all four structural motifs. The occupancies for the surface atoms representing the four motifs, FlatStoichiometric, FlatVacancy, RumpledStoichiometric, and RumpledVacancy $\left(x_{\mathrm{FS}}, x_{\mathrm{FV}}, x_{\mathrm{RS}}\right.$, and $x_{\mathrm{RV}}$, respectively), were constrained such that the sum of the four occupancies is 1 , and initially each motif was assigned an occupancy of $25 \%$.

A $\mathrm{TiO}_{2}$ unit was placed on top of the surface's zigzag with occupancy $x_{\mathrm{TiO}_{2}}$ to correlate with the bright protrusions in the experimental STM images. Owing to the symmetry constraints of the refinement, the $\mathrm{TiO}_{2}$ was added in a periodic fashion, because adding a single $\mathrm{TiO}_{2}$ unit in the unit cell would require a reduction in the symmetry, therefore increasing the number of parameters $(p)$, which is undesirable. Thus, to model the nonperiodic nature of the $\mathrm{TiO}_{2}$ unit, the occupancy $\left(x_{\mathrm{TiO}_{2}}\right)$ was allowed to vary as an independent variable.

Table I shows the figures of merit for each of the structural refinements: four motifs combined plus the $\mathrm{TiO}_{2}$ unit, four motifs combined without $\mathrm{TiO}_{2}$ unit, RumpledStoichiometric, FlatStoichiometric, FlatVacancy, and RumpledVacancy. It is important to note that the positions relaxed by the DFT calculations did not change much upon refinement, providing strong evidence that they are appropriate models. Using the Hamilton $R$-factor ratio, ${ }^{41}$ the structure with the four motifs combined fits the data better than any of the other individual models with greater than 90\% certainty. Other models were tested, including structures composed of combinations of two or three of the structural motifs and structures incorporating the DoubleVacancy motif; however, these refinements tended to be inferior and supported the four structural motif model. 
TABLE I. Figures of merit for refinement of DFT-relaxed structures against SXRD data.

\begin{tabular}{lccccc}
\hline \hline Model & $\begin{array}{c}\text { No. of } \\
\text { LS }\end{array}$ & $\begin{array}{c}\text { No. of } \\
\text { data }(n)\end{array}$ & $\begin{array}{c}\text { No. of } \\
\text { parameters }(m)\end{array}$ & $w R^{2}$ & $\begin{array}{c}\text { Goodness } \\
\text { of fit }\end{array}$ \\
\hline Four motifs combined, with $\mathrm{TiO}_{2}$ unit & 25 & 848 & 286 & 0.65 & 5.67 \\
Four motifs combined, without $\mathrm{TiO}_{2}$ unit & 25 & 848 & 280 & 0.65 & 5.65 \\
RumpledStoichiometric only & 25 & 848 & 158 & 0.74 & 6.27 \\
FlatStoichiometric only & 25 & 848 & 158 & 0.74 & 6.33 \\
RumpledVacancy only & 25 & 848 & 157 & 0.77 & 6.69 \\
FlatVacancy only & 25 & 848 & 157 & 0.73 & 6.25 \\
\hline \hline
\end{tabular}

The figures of merit for the individual structure refinements are similar for the FlatVacancy, RumpledStoichiometric, and FlatStoichiometric structures and showed a worse fit for the RumpledVacancy structure, all in qualitative agreement with the relative surface energy values. For the four motifs combined structure, the final values for $x_{\mathrm{FS}}, x_{\mathrm{FV}}, x_{\mathrm{RS}}$, and $x_{\mathrm{RV}}$ each remained close to $25 \%$, i.e., each structural motif is present on approximately $\frac{1}{4}$ of the surface. The $\mathrm{TiO}_{2}$ unit $\left(x_{\mathrm{TiO}_{2}}\right)$ is situated on roughly $15 \%-45 \%$ of the $c(6 \times 2)$ surface unit cells, which agrees well with the experimental STM measurement of approximately $33 \%$. Data from the second sample, also annealed in $\mathrm{O}_{2}$ at $1100{ }^{\circ} \mathrm{C}$ but subsequently annealed in UHV at $300{ }^{\circ} \mathrm{C}$, also gave similar occupancies for $x_{\mathrm{FS}}, x_{\mathrm{FV}}, x_{\mathrm{RS}}, x_{\mathrm{RV}}$, and $x_{\mathrm{TiO}_{2}}$ in the four motifs combined structure, which is expected since the oxygen chemical potential at $1000{ }^{\circ} \mathrm{C}$ in $\mathrm{O}_{2}$ and at $300{ }^{\circ} \mathrm{C}$ in $\mathrm{UHV}$ are similar $(-3.2$ and $-2.57 \mathrm{eV}$, respectively).

\section{DISCUSSION}

A model for the structure of the $c(6 \times 2)$ reconstruction has been proposed, and unlike the $(2 \times 1)$ and $c(4 \times 2)$ reconstructions on $\mathrm{SrTiO}_{3}(001)$, the $c(6 \times 2)$ structure solution was not explicitly provided from direct methods analysis alone. Of the three reconstructions, the $c(6 \times 2)$ forms at the highest temperature, $1050-1100{ }^{\circ} \mathrm{C}$, compared to $850-930{ }^{\circ} \mathrm{C}$ for $c(4 \times 2)$ and $950-1050{ }^{\circ} \mathrm{C}$ for $(2 \times 1)$, and is therefore, not surprisingly, the most complex structure. The surface is composed of short-range-ordered domains of four related structures, ranging from stoichiometric to slightly reduced (one oxygen vacancy per primitive surface unit cell), each present on approximately $\frac{1}{4}$ of the total surface area. At the temperature and oxygen partial pressure required for the formation of the $c(6 \times 2)$ surface reconstruction, the formation energies for these structures are quite comparable, and the surface thus takes the form of a random (although short-range-ordered) mixture of these four structural motifs. A rough approximation for the entropy of mixing is $1.39 \mathrm{kT}$ per unit cell area, which at $1100{ }^{\circ} \mathrm{C}$ is $0.164 \mathrm{eV}$. This value represents the upper bound, as it neglects domain boundary energy and assumes that the structure of one unit cell does not influence the structure of neighboring cells. Additionally, the $\mathrm{TiO}_{2}$ unit, which is present nonperiodically on the surface, also results in an entropic free energy gain for the surface.
The proposed $c(6 \times 2)$ structure, while the most complicated reconstruction on $\mathrm{SrTiO}_{3}$, shows similarities to the $(2$ $\times 1)$ and $c(4 \times 2)$ structures. ${ }^{7,8}$ All three structures are terminated with a $\mathrm{Ti}_{y} \mathrm{O}_{x}$ surface layer, that is, there are no strontium atoms on the surface. The $c(4 \times 2)$ and $(2 \times 1)$ reconstructions are composed of a single $\mathrm{TiO}_{2}$-stoichiometry overlayer above bulklike $\mathrm{TiO}_{2}$, and the difference between the $c(4 \times 2)$ and $(2 \times 1)$ structures is the distribution of the surface $\mathrm{Ti}$ among the possible sites. The $c(6 \times 2)$, on the other hand, has a thicker (more than one) $\mathrm{TiO}_{x}$ overlayer above the bulklike $\mathrm{TiO}_{2}$ layer. Furthermore, the $c(4 \times 2)$ and $(2 \times 1)$ structures have titanium cations present on the surface solely in the form of fivefold, truncated octahedra, and while the $c(6 \times 2)$ reconstruction does have titanium cations in fivefold truncated octahedra, titanium cations are also present in the surface structure with fourfold coordination. The most striking difference is the fact that the $c(6 \times 2)$ reconstruction is composed of multiple related, but different, structural domains, while the $c(4 \times 2)$ and $(2 \times 1)$ reconstructions are single-structure surfaces. Finally, $\mathrm{TiO}_{2}$ units are stabilized on the surface of $\mathrm{SrTiO}_{3}(001)-c(6 \times 2)$, but no evidence exists for this type of behavior on the $c(4 \times 2)$ or $(2 \times 1)$ surfaces.

It is believed that the $c(6 \times 2)$ surface is likely to be the most catalytically active surface of $\mathrm{SrTiO}_{3}(001)$. With titanium atoms present in multiple coordination geometries and oxidation states, the surface would likely be able to bind reactant molecules and promote redox-type reactions. The $c(6 \times 2)$ reconstruction $[$ as well as the $(2 \times 1)]$ contains $\mathrm{Ti}=\mathrm{O}$ (titanyl) groups which have recently been implicated with catalytic activity on the (011) surface of rutile $\mathrm{TiO}_{2} \cdot{ }^{42}$ Furthermore, the presence of the $\mathrm{TiO}_{2}$ unit suggests the ability of the surface to stabilize reaction intermediates, and research is currently under way to investigate the adsorption, desorption, and reactivity of methyl radicals on the various reconstructions of $\mathrm{SrTiO}_{3}$ (001).

\section{CONCLUSIONS}

In conclusion, a model for the atomic-scale structure of the $\mathrm{SrTiO}_{3}(001)-c(6 \times 2)$ surface reconstruction has been proposed. The surface reconstruction is formed at high temperatures $\left(1050-1100{ }^{\circ} \mathrm{C}\right)$ in oxidizing conditions and is highly stable and reproducible. The surface is composed of domains of similar but distinct structures, and additionally, 
$\mathrm{TiO}_{2}$ units are randomly distributed on the surface. While the structure solution method was not conventional, we have acquired the maximum amount of information through a combination of techniques. Transmission electron diffraction and surface $\mathrm{x}$-ray diffraction provided the positions of the surface titanium atoms averaged over the four structural motifs, and the $a b$ initio screening technique proved to be indispensable for the determination of oxygen positions, as well as the titanium positions along the $z$ direction. Adaptation of the SHELX-97 program for structure refinement against surface $\mathrm{x}$-ray data merged theory with experiment to corroborate the model, and finally STM simulations confirmed consistency with experimental observations.

\section{ACKNOWLEDGMENTS}

C.H.L., K.R.P., and L.D.M. were supported by the Chemical Sciences, Geosciences and Biosciences Division, Office of Basic Energy Sciences, U.S. Department of Energy Office of Science Grant No. DE-FG02-03ER15457, and N.E. and O.W. were supported by the EMSI program of the National Science Foundation and the U.S. Department of Energy Office of Science Grant No. CHE-9810378, all at the Northwestern University Institute for Environmental Catalysis. A.v.d.W and M.A. were supported by the National Science Foundation under program NSF-MRSEC (DMR-00706097) and through TeraGrid computing resources provided by NCSA and SDSC. E.L. was supported by the National Science Foundation via Grant No. DMR-9214505. A.K. and J.Z. were supported by the German BMBF under Contracts Nos. 05SE8GUA5 and 05KS1GUC3. The work of A. v. d. W., N. E., E. L., and M. A. was performed while at Northwestern University, Department of Materials Science and Engineering. The work of O.W. was performed while at Northwestern University, Department of Physics and Astronomy. The work of A. K. was performed while at Max-Planck-Institute für Festkörperforschung.
${ }^{1}$ V. E. Henrich and P. A. Cox, The Surface Science of Metal Oxides (Cambridge University Press, Cambridge, 1994).

${ }^{2}$ Epitaxial Oxide Thin Films II, edited by J. S. Speck, D. K. Fork, R. M. Wolf, and T. Shiosaki, MRS Symposia Proceedings No. 401 (Materials Research Symposia, Pittsburgh, PA, 1996).

${ }^{3}$ R. A. McKee, F. J. Walker, and M. F. Chisholm, Phys. Rev. Lett. 81, 3014 (1998).

${ }^{4}$ R. A. McKee, F. J. Walker, and M. F. Chisholm, Science 293, 468 (2001).

${ }^{5}$ J. G. Mavroides, J. A. Kafalas, and D. F. Kolesar, Appl. Phys. Lett. 28, 241 (1976).

${ }^{6}$ B. Cord and R. Courths, Surf. Sci. 162, 34 (1985).

${ }^{7}$ N. Erdman, K. R. Poeppelmeier, M. Asta, O. Warschkow, D. E. Ellis, and L. D. Marks, Nature (London) 419, 55 (2002).

${ }^{8}$ N. Erdman, O. Warschkow, M. Asta, K. R. Poeppelmeier, D. E. Ellis, and L. D. Marks, J. Am. Chem. Soc. 125, 10050 (2003).

${ }^{9}$ T. Nishimura, A. Ikeda, H. Namba, T. Morishita, and Y. Kido, Surf. Sci. 421, 273 (1999).

${ }^{10}$ V. Vonk, S. Konings, G. J. van Hummel, S. Harkema, and H. Graafsma, Surf. Sci. 595, 183 (2005).

${ }^{11}$ Q. D. Jiang and J. Zegenhagen, Surf. Sci. 338, L882 (1995).

${ }^{12}$ T. Matsumoto, H. Tanaka, T. Kawai, and S. Kawai, Surf. Sci. 278, L153 (1992).

${ }^{13}$ P. J. Moller, S. A. Komolov, and E. F. Lazneva, Surf. Sci. 425, 15 (1999).

${ }^{14}$ H. Tanaka, T. Matsumoto, T. Kawai, and S. Kawai, Jpn. J. Appl. Phys., Part 1 32, 1405 (1993).

${ }^{15}$ T. Kubo and H. Nozoye, Phys. Rev. Lett. 86, 1801 (2001).

${ }^{16}$ M. S. M. Gonzalez, M. H. Aguirre, E. Moran, M. A. AlarioFranco, V. Perez-Dieste, J. Avila, and M. C. Asensio, Solid State Sci. 2, 519 (2000).

${ }^{17}$ M. Naito and H. Sato, Physica C 229, 1 (1994).

${ }^{18}$ T. Kubo and H. Nozoye, Surf. Sci. 542, 177 (2003).

${ }^{19}$ M. R. Castell, Surf. Sci. 516, 33 (2002).

${ }^{20}$ K. Johnston, M. R. Castell, A. T. Paxton, and M. W. Finnis, Phys. Rev. B 70, 085415 (2004).
${ }^{21}$ O. Warschkow, M. Asta, N. Erdman, K. R. Poeppelmeier, D. E. Ellis, and L. D. Marks, Surf. Sci. 573, 446 (2004).

${ }^{22}$ L. M. Liborio, C. G. Sanchez, A. T. Paxton, and M. W. Finnis, J. Phys.: Condens. Matter 17, L223 (2005).

${ }^{23}$ Q. D. Jiang and J. Zegenhagen, Surf. Sci. 367, L42 (1996).

${ }^{24}$ Q. D. Jiang and J. Zegenhagen, Surf. Sci. 425, 343 (1999).

${ }^{25}$ L. D. Marks, E. Bengu, C. Collazo-Davila, D. Grozea, E. Landree, C. Leslie, and W. Sinkler, Surf. Rev. Lett. 5, 1087 (1998).

${ }^{26}$ L. D. Marks, N. Erdman, and A. Subramanian, J. Phys.: Condens. Matter 13, 10677 (2001).

${ }^{27}$ X. Torrelles, J. Zegenhagen, J. Rius, T. Gloege, L. X. Cao, and W. Moritz, Surf. Sci. 589, 184 (2005).

${ }^{28}$ C. H. Lanier, J. M. Rondinelli, B. Deng, R. Kilaas, K. R. Poeppelmeier, and L. D. Marks, Phys. Rev. Lett. 98086102 (2007).

${ }^{29}$ G. M. Sheldrick (unpublished).

${ }^{30}$ See EPAPS Document No. E-PRBMDO-76-051728 for a copy of SXRD data, a copy of input (.ins) file and final $\left(F_{c}\right)^{2}$ values, the atomic positions of the four structural motifs, and the DFT refined positions for $\mathrm{TiO}_{2}$ unit. For more information on EPAPS, see http://www.aip.org/pubservs/epaps.html.

${ }^{31}$ G. Kresse and J. Hafner, Phys. Rev. B 47, 558 (1993).

${ }^{32}$ G. Kresse and J. Hafner, Phys. Rev. B 49, 14251 (1994).

${ }^{33}$ G. Kresse and J. Furthmuller, Comput. Mater. Sci. 6, 15 (1996).

${ }^{34}$ G. Kresse and J. Furthmuller, Phys. Rev. B 54, 11169 (1996).

${ }^{35}$ D. Vanderbilt, Phys. Rev. B 41, 7892 (1990).

${ }^{36}$ G. Kresse and J. Hafner, J. Phys.: Condens. Matter 6, 8245 (1994).

${ }^{37}$ D. M. Ceperley and B. J. Alder, Phys. Rev. Lett. 45, 566 (1980).

${ }^{38}$ J. Tersoff and D. R. Hamann, Phys. Rev. B 31, 805 (1985).

${ }^{39}$ N. Erdman and L. D. Marks, Surf. Sci. 526, 107 (2003).

${ }^{40}$ M. W. Chase, C. A. Davies, J. R. Downey, D. J. Frurip, R. A. McDonald, and A. N. Syverud, J. Phys. Chem. Ref. Data 14, 927 (1985).

${ }^{41}$ W. C. Hamilton, Acta Crystallogr. 18, 502 (1965).

${ }^{42}$ T. J. Beck, A. Klust, M. Batzill, U. Diebold, C. Di Valentin, and A. Selloni, Phys. Rev. Lett. 93, 036104 (2004). 